\title{
Étude des premiers événements contribuant à l'implantation des cellules cancéreuses dans une niche métastatique
}

\author{
Claire Marceaux ${ }^{1}$, Marie-Liesse Asselin-Labat ${ }^{1,2}$
}

$>$ Le processus à l'origine de la migration des cellules cancéreuses dans un organe afin de former des métastases est finement contrôlé et nécessite de nombreuses modifications cellulaires. $\varepsilon n$ effet, ces cellules doivent pénétrer et survivre dans la circulation sanguine, sortir des vaisseaux sanguins, pour finalement coloniser un nouvel organe [1]. Pour subsister dans l'organe hôte, les cellules métastatiques s'adaptent et modifient leur niche cellulaire. II est important de caractériser cette niche cellulaire afin de comprendre les évènements responsables de la formation des métastases et d'identifier de nouvelles stratégies thérapeutiques.

\section{Méthodes pour étudier la niche} métastatique in vivo

Les évènements moléculaires et cellulaires à l'origine de l'implantation d'une cellule cancéreuse dans un nouveau microenvironnement sont restés longtemps peu étudiés à cause du manque d'outils pour évaluer ces changements in vivo. De nouvelles techniques ont récemment été développées pour étudier spécifiquement in vivo les cellules qui forment la niche dans laquelle les cellules tumorales métastatiques viennent s'implanter. Ces techniques utilisent des marqueurs fluorescents permettant de visualiser et d'isoler les différents types cellulaires présents dans la niche (Figure 1).

Les cellules tumorales secrètent constamment des facteurs solubles ou des vésicules extracellulaires (extracellular vesicles, عV) qui transportent des molécules spécifiques affectant les fonctions des cellules receveuses [2]. Ce système de transport par les $E V$ a été exploité par Zomer et al. pour marquer les cellules de la niche métastatique in vivo [3]. Leur méthode, fondée sur l'utilisation du système Cre-loxP, consiste à modifier les cellules cancéreuses donneuses pour qu'elles expriment la recombinase Cre, dont I'ARNm est sécrété dans leurs $\varepsilon V$. En parallèle, les cellules receveuses sont modifiées génétiquement pour exprimer une protéine rapporteur fluorescente seulement après recombinaison de l'allèle correspondant par l'enzyme Cre. Les cellules ayant invaginé des EV (contenant I'ARNm codant (re) provenant des cellules métastatiques deviennent alors fluorescentes. Cette méthode permet d'analyser les cellules affectées par les cellules donneuses en les comparant directement avec les cellules n'ayant pas incorporé d'EV (Figure IA). Les auteurs ont montré que l'expression de la protéine Cre dans les cellules receveuses ne se faisait que par transfert de son ARNm présent dans les $\varepsilon V$, et non lorsque la protéine était sécrétée sous forme libre. Cette approche permet d'étudier les communications cellulaires in vitro, mais également in vivo, à proximité ou à distance de la tumeur primaire, au niveau du site métastatique, grâce à l'imagerie réalisée directement sur l'animal vivant.

Plus récemment, 0 mbrato et al. [4] ont décrit une méthode permettant de détecter les cellules situées dans le proche environnement des cellules
${ }^{1}$ Personalised Oncology Division, The Walter and Eliza Hall Institute of Medical Research, Department of Medical Biology, The University of Melbourne, Parkville, Australie.

${ }^{2}$ Cancer Early Detection and Advanced Research Center, Knight Cancer Institute, Oregon Health and Science University, Portland, États-Unis.

labat@wehi.edu.au

métastatiques par un marquage passif n'impliquant pas le transfert via les $\varepsilon V$. Cette méthode consiste à modifier génétiquement des cellules de cancer du sein pour qu'elles expriment une molécule liposoluble attachée à une protéine fluorescente mCherry (sLP-mCherry). Cette molécule est sécrétée librement ou dans les EV des cellules tumorales, et pénètre passivement dans les cellules adjacentes à travers leur membrane plasmique (Figure 1B). Ombrato et al. ont montré que seules les protéines libres, et non les protéines présentes dans les $\varepsilon V$, pénétraient dans les cellules proches, offrant ainsi une méthode différente de celle décrite par Zomer et al. La sLP-mCherry est stable dans les cellules receveuses pendant environ 48 heures, ce qui facilite l'analyse des premiers évènements se déroulant dans les cellules de la niche, mais ne permet pas une étude des changements cellulaires sur le long terme. Cette méthode de visualisation par microscopie des cellules formant la niche cellulaire permet la localisation précise de cette dernière, et met en évidence les interactions intercellulaires qui s'y produisent. De plus, la présence d'un fluorochrome permet, après dissociation du tissu hôte, d'isoler des cellules composant la niche par cytométrie en flux, pour des analyses moléculaires (e.g., RNAseq) et fonctionnelles.

Ces deux méthodes de marquage cellulaire à partir des cellules cancéreuses présentent des avantages différents. La technique Cre-loxP (Figure 1A) [3] permet d'analyser sur le long terme les différents 
A

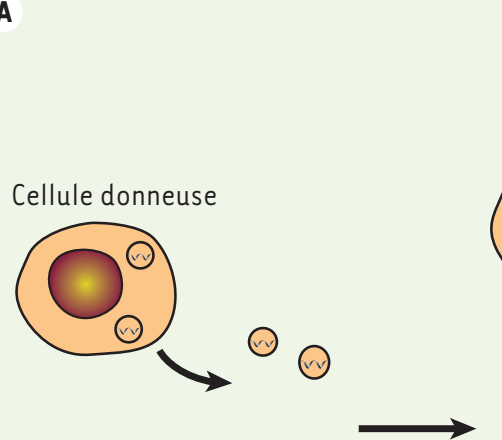

Cellule receveuse

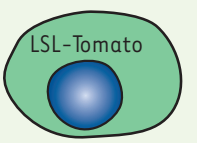

À proximité

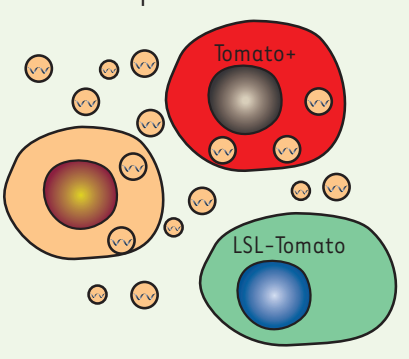

À distance

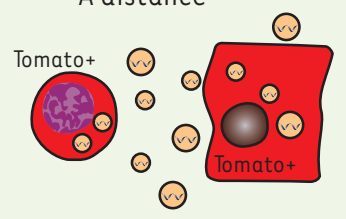

B

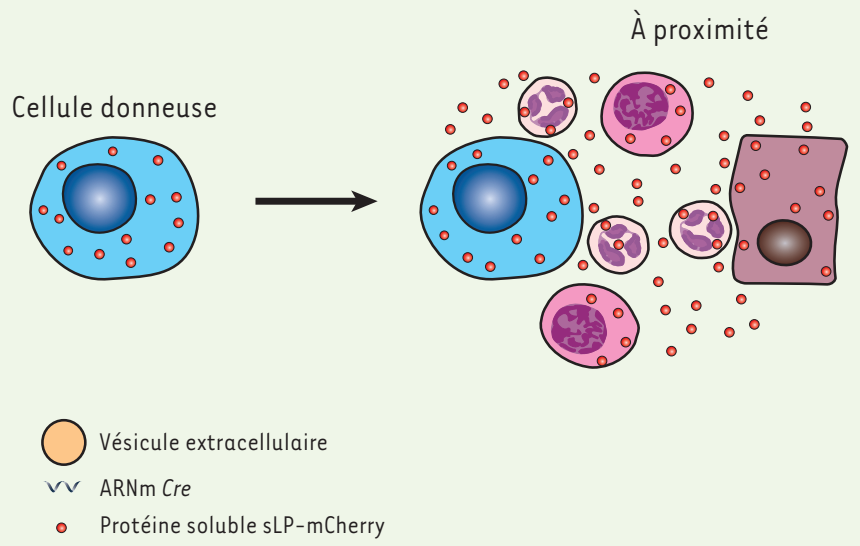

Figure 1. Méthodes de marquage in vivo des cellules de la niche métastatique situées au voisinage des cellules tumorales. $A$. Les cellules cancéreuses donneuses (orange) sécrètent des vésicules extracellulaires $(\varepsilon V)$ contenant l'ARNm codant la recombinase Cre. L'incorporation de ces $\varepsilon V$ par des cellules receveuses (vertes) génétiquement modifiées pour exprimer un fluorochrome exclusivement après recombinaison par l'enzyme Cre, se fait soit à proximité soit à distance des cellules donneuses, et produit des cellules acceptrices fluorescentes (rouges), ce qui facilite leur étude. LSL-Tomato : Lox-STOP-Lox-Tomato cassette. B. Les cellules cancéreuses métastatiques donneuses (bleues) expriment des molécules liposolubles fluorescentes (sLP-mCherry, en rouge), qui sont incorporées, par diffusion passive, dans les cellules adjacentes aux cellules cancéreuses.

évènements moléculaires induits par les cellules tumorales à distance de la tumeur primaire, au site métastatique. La méthode utilisant une protéine libre liposoluble (Figure 1B) [4] facilite, quant à elle, l'analyse des premiers évènements qui ont lieu dans la niche métastatique, leur croissance, notamment en augmentant l'angiogenèse afin de recevoir plus de nutriments et d'oxygène, ou en exprimant des molécules bloquant leur reconnaissance par les cellules du système immunitaire [5]. De façon similaire, les cellules qui nichent dans un nouveau microenvironnement pour former une métastase altèrent les cellules normales autour d'elles, par exemple en limitant leur accès aux métabolites nutritifs, au profit des cellules métastatiques [6]. Plusieurs études ont montré le rôle des cellules de la lignée myéloïde $\left(C D 11 b^{+}\right)$pour préparer une niche métastatique et favoriser la croissance de cellules tumorales métastatiques dans le poumon [7-10]. La méthode développée par 0mbrato et al. [4] a désormais permis de montrer l'effet direct des cellules métastatiques sur les cellules épithéliales normales du poumon pour promouvoir la croissance des cellules cancéreuses (Figure 2).

Les monocytes et les macrophages jouent un rôle important dans la formation de la niche métastatique. Ainsi, la déplétion des macrophages du poumon réduit la migration des cellules du cancer du sein et l'implantation de métastases pulmonaires [7]. L'infiltration de polynucléaires neutrophiles dans les tumeurs est également associée à un mauvais pronostic de survie des patients. Une méta-analyse a suggéré que la proportion de neutrophiles par rapport aux lymphocytes dans les tumeurs solides constitue un biomarqueur de survie [8]. Des études dans des modèles précliniques ont en effet démontré le rôle des neutrophiles pour préparer une niche pré-métastatique et promouvoir les métastases pulmonaires du cancer du sein $[9,10]$. En utilisant leur méthode de marquage avec sLPmCherry (Figure 1B), Ombrato et al. ont démontré in situ la présence des neutrophiles dans la niche métastatique dès l'implantation des premières cellules cancéreuses disséminées par voie sanguine. Une augmentation des dérivés réactifs de l'oxygène (reactive 
A

Poumon sain

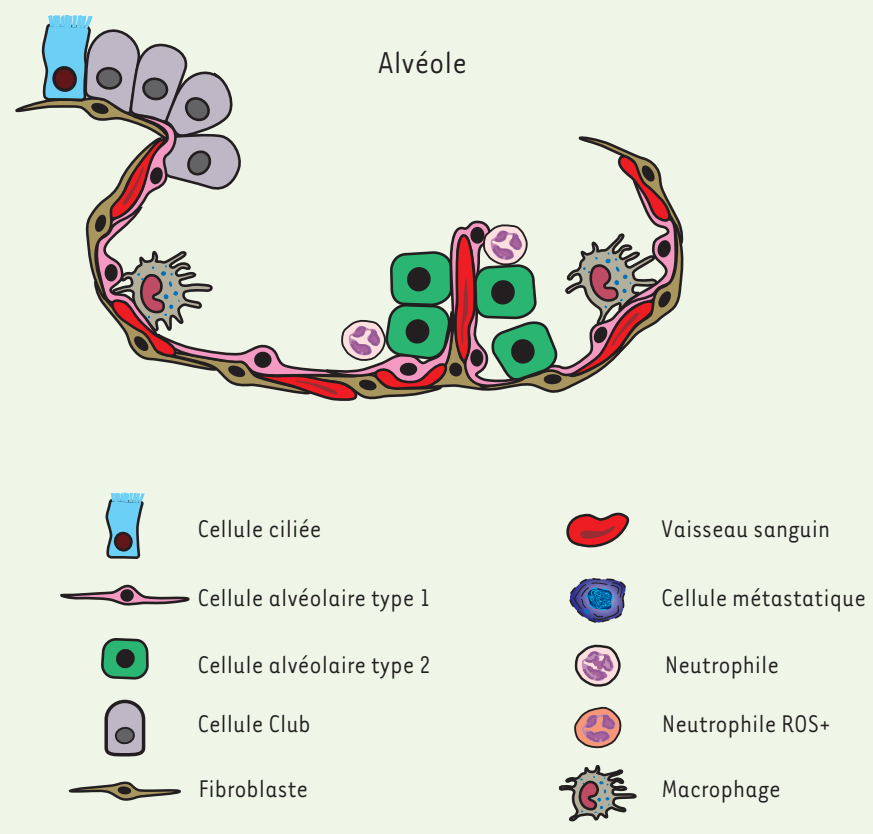

Niche métastatique
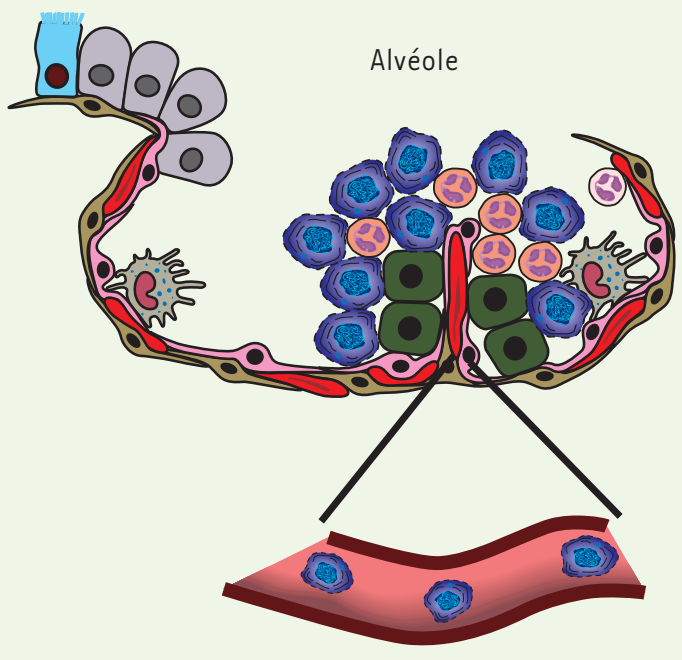

Figure 2. Changements cellulaires dans la niche métastatique. A. Schéma de l'organisation des alvéoles pulmonaires saines. B. L'implantation des cellules métastatiques, après migration dans les vaisseaux sanguins, entraîne notamment le recrutement de polynucléaires neutrophiles exprimant de grandes quantités de dérivés réactifs de l'oxygène (reactive oxygen species, ROS) et modifie les propriétés des cellules alvéolaires environnantes, ce qui favorise l'implantation et la prolifération des cellules métastatiques.

oxygen species, ROS) a été observée dans les neutrophiles présents dans la niche métastatique, par rapport aux neutrophiles pulmonaires présents à distance de la métastase. Cette augmentation des ROS, connue pour activer une réponse inflammatoire pro-tumorale, a aussi été retrouvée dans des modèles murins de cancer du poumon, où elle favorise la croissance tumorale [11]. La co-culture de polynucléaires neutrophiles avec des cellules tumorales in vitro augmente leur prolifération, mais ce phénomène est bloqué en présence d'un inhibiteur de ROS, prouvant l'importance de ces ROS produites par les neutrophiles pour la croissance des métastases [4].

Les cellules métastatiques affectent non seulement les cellules du système immunitaire, mais aussi les propriétés des autres cellules tumorales et des cellules épithéliales normales. En effet, Zomer et al. ont montré que l'invagination des EV sécrétées par des cellules métastatiques, par des cellules tumorales non métastatiques augmente leur potentiel migratoire et invasif. De plus, Liu et al. ont démontré que les $\varepsilon V$ des cellules cancéreuses modulent à distance les cellules épithéliales normales du poumon, induisant la sécrétion par les cellules alvéolaires de facteurs permettant le recrutement des neutrophiles pro-inflammatoires et la préparation de la niche pré-métastatique [9]. Une fois installées dans le poumon, les cellules du cancer du sein ont la capacité de modifier les cellules épithéliales situées autour d'elles, leur conférant des propriétés similaires à celles des cellules souches épithéliales du poumon. Ces cellules épithéliales provoquent alors la prolifération des cellules cancé- reuses, ce qui favorise la croissance de la tumeur métastatique [4].

Ces différentes études et le développement de nouvelles méthodes pour identifier et isoler les cellules affectées par les cellules cancéreuses, à proximité ou à distance de celles-ci, permettent une meilleure compréhension de l'impact des cellules cancéreuses sur leur environnement.

\section{Implications thérapeutiques}

Compte tenu de l'instabilité génétique des cellules tumorales et de leur capacité à s'adapter rapidement à leur environnement pour proliférer, migrer ou adopter des moyens de résistance aux thérapies, cibler les cellules normales autour de la tumeur primitive ou de sa métastase peut constituer une stratégie de traitement. Des médicaments bloquant l'angiogenèse ou activant les 
cellules du système immunitaire ont déjà prouvé l'intérêt d'une telle stratégie. Étant donné que les décès des patients atteints d'un cancer sont la plupart du temps causés par les métastases, bloquer la migration et l'implantation des cellules métastatiques dans la niche métastatique est une nouvelle possibilité thérapeutique à explorer. Cependant, il reste beaucoup à apprendre sur le microenvironnement des cellules métastatiques et la manière dont les cellules cancéreuses manipulent cet environnement, à distance avant leur implantation, et une fois arrivées sur le site de la métastase. Les méthodes de marquage des cellules de la niche métastatique développées par Zomer et al. [3], et plus récemment par 0 mbrato et al. [4], offrent de nouvelles perspectives pour comprendre les communications entre les cellules cancéreuses et non cancéreuses voisines, et identifier ainsi de nouvelles cibles thérapeutiques. $\diamond$ Study of early events influencing the settlement of cancer cells in metastatic niches

\section{LIENS D'INTÉRÊT}

Les auteures déclarent n'avoir aucun lien d'intérêt concernant les données publiées dans cet article.

\section{RÉFÉRENCES}

1. Quail DF, Joyce JA. Microenvironmental regulation of tumor progression and metastasis. Nat Med 2013; 19 : 1423-37.

2. Raposo G, Stoorvogel W. Extracellular vesicles: exosomes, microvesicles, and friends. J Cell Biol 2013; $200: 373-83$.

3. Zomer A, Maynard C, Verweij FJ, et al. In vivo imaging reveals extracellular vesicle-mediated phenocopying of metastatic behavior. Cell 2015 ; 161 : 1046-57.

4. Ombrato L, Nolan $\varepsilon$, Kurelac I, et al. Metastatic- niche labelling reveals parenchymal cells with stem features. Nature $2019 ; 572: 603-8$.

5. Hanahan D, Weinberg RA. Hallmarks of cancer: the next generation. Cell $2011 ; 144: 646-74$.

6. Fong MY, Zhou W, Liu L, et al. Breast-cancersecreted miR-122 reprograms glucose metabolism in premetastatic niche to promote metastasis. Nat Cell Biol 2015; 17 : 183-94.

7. Linde N, Casanova-Acebes M, Sosa MS, et al. Macrophages orchestrate breast cancer early dissemination and metastasis. Nat Commun 2018; $9: 21$.

8. Templeton AJ, McNamara MG, Šeruga B, et al. Prognostic role of neutrophil-to-lymphocyte ratio in solid tumors: a systematic review and meta-analysis. J Natl Cancer Inst 2014 ; 106 : djul24.

9. Liu Y, Gu Y, Han Y, et al. Tumor exosomal RNAs promote lung pre-metastatic niche formation by activating alveolar epithelial TLR3 to recruit neutrophils. Cancer Cell 2016 ; 30 : 243-56.

10. Wculek SK, Malanchi I. Neutrophils support lung colonization of metastasis-initiating breast cancer cells. Nature $2015 ; 528: 413-17$.

11. Engblom C, Pfirschke C, Zilionis R, et al. Osteoblasts remotely supply lung tumors with cancer-promoting SiglecF-high neutrophils. Science 2017 ; 358 : eaal5081. 